\title{
Orbits versus Star Formation Histories: A Progress Report
}

\author{
Eva K. Grebel \\ UCO/Lick Obs., University of California, Santa Cruz, CA 95064, USA \\ Tsafrir Kolatt \\ Physics Dept., University of California, Santa Cruz, CA 95064, USA \\ Wolfgang Brandner \\ JPL/IPAC, Mail Code 100-22, Pasadena, CA 91125, USA
}

\begin{abstract}
Milky Way dwarf companions and satellites of distant spirals seem to show a preference for polar orbits. We suggest that five out of six M31 dwarf spheroidal companions as well as two dwarf irregulars may also be located near a polar plane.

We briefly discuss preliminary results from a statistical approach to study correlations between star formation histories and orbits of Local Group dwarf galaxies, such as a possible correlation between star formation episodes and galaxy separations.
\end{abstract}

\section{Polar Orbits Preferred?}

Dwarf galaxies are believed to have been more numerous at earlier times but to have been largely accreted since then by larger galaxies. Hierarchical merger scenarios suggest that they contributed significantly to the build-up of more massive galaxies in galaxy groups and clusters. Galaxies on polar orbits are more stable against accretion. Many of the satellite galaxies observed today may be on polar or near-polar orbits ensuring their survival over a longer time period. Zaritsky et al. (1997) found a tendency for higher concentrations of satellite galaxies in the polar regions of distant massive spirals. The future of galaxy groups and clusters may be continued accretion until the dwarf supply is used up.

The Milky Way's dwarf satellites and a number of halo globular clusters appear to lie along two (e.g., Majewski 1994) or more (e.g., Lynden-Bell \& Lynden-Bell 1995) polar great circles, possibly indicating a common origin as remnants of a larger parent galaxy since accreted by the Milky Way.

Do the satellites of the most massive spiral galaxy in the Local Group, M31, also show indications of one (or several) preferred polar orbital planes? 

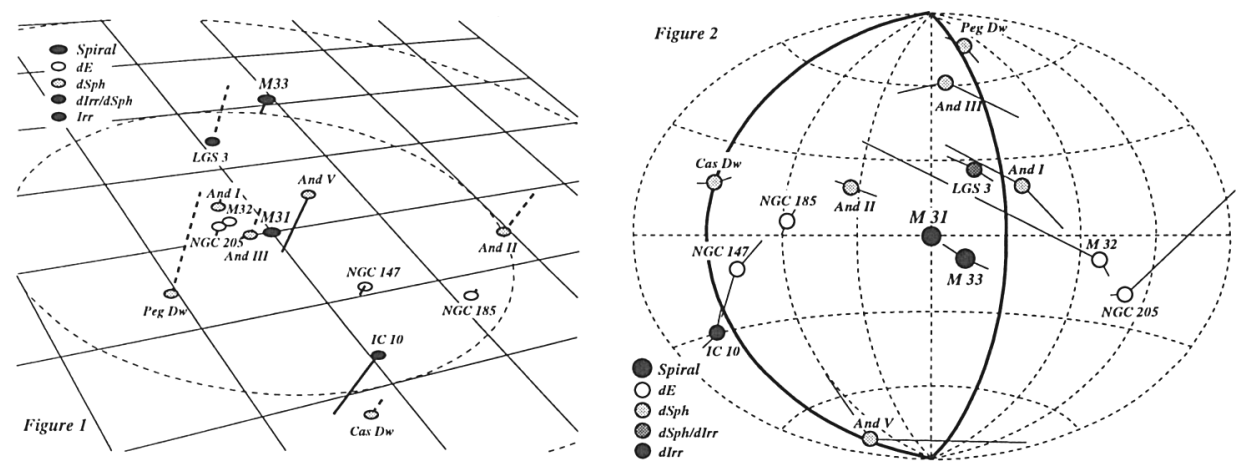

Figure 1. A 3-D representation of M31 and its satellite system in the M31 plane (grid). Each side of a square in the grid has a length of 100 kpc. The dashed circles mark radii of 200 and $400 \mathrm{kpc}$. Straight lines combining the galaxies with the plane represent their actual distance above or below (dashed) the plane. Morphological separation of the satellite galaxies with distance from M31 is evident.

Figure 2. Aitoff projection of the M31 satellite system in a native $l_{M 31}, b_{M 31}$ coordinate system. Thin solid lines indicate the positional uncertainties resulting from poorly known distances. A potential polar orbital plane is indicated by the bold line. Most of the M31 dSph companions are found near this "Andromeda great circle".

\section{M31: A Polar Great Circle of Satellites?}

We use distance determinations and coordinates of M31 and its companions to search for potential polar great circles of satellites around M31. Starting from $l, b$, the distance moduli of M31 and its satellites as well as the orientation and inclination of the disk of M31, we defined a native $l_{M 31}$ and $b_{M 31}$ coordinate system. The coordinates of the M31 satellites were transformed into the new system. For galaxies close to M31 (i.e., at distances $<100 \mathrm{kpc}$ ) the placement in the new coordinate system is quite uncertain since the relative and absolute distances between and to M31 and its satellites are poorly known. We adopted the Cepheid distance of $770 \mathrm{kpc}$ (Freedman \& Madore 1990) for M31. The resulting 3-D distribution of galaxies around M31 is shown in Fig. 1.

Neglecting distance uncertainties, we find that the resulting Aitoff projection (Fig. 2) shows most of M31's dSph companions near a polar plane: And I, III, V, Peg Dw, Cas Dw as well as the dIrr/dSph LGS 3 and dIrr IC 10 may form a potential "Andromeda great circle" (see also Grebel 1998). NGC 147 lies close to the assumed plane. Even the much more distant M33 appears to be near this plane. And II and the dEs M32, NGC 185, and NGC 205 do not lie close to the suggested polar plane but are all located near the equatorial plane of M31. We note that van den Bergh (1998, based on different distances) suggests that NGC 147 forms a binary system with NGC 185. If the 13 galaxies were distributed at random, the probability of finding 7 galaxies within $\pm 15^{\circ}$ of a great circle is $\leq 5 \%$. 


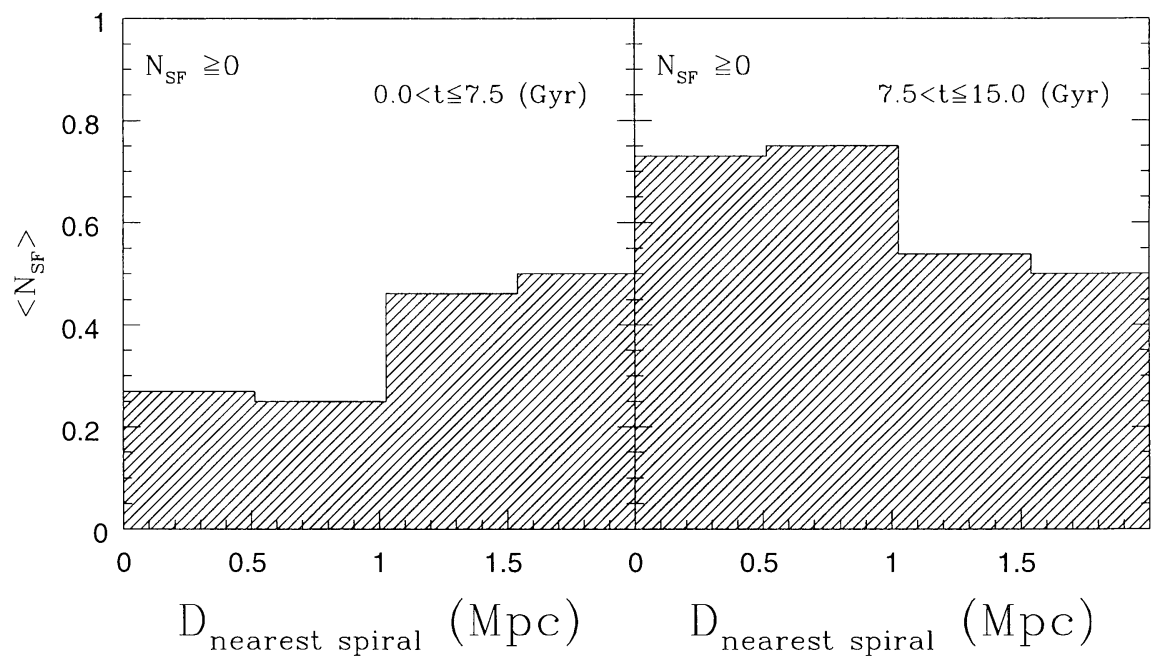

Figure 3. Examples for the statistical approach. Average number of star formation episodes for galaxies with more than one episode versus distance from the nearest spiral (uncorrected for population detectability bias). Star formation episodes that started less (more) than $7.5 \mathrm{Gyr}$ ago are shown on the left (right).

At present there are no radial velocity measurements for any of the dSphs around M31, nor do we have any other orbital information. H I surveys did not show the presence of gas streams similar to the Magellanic Stream (Brinks, priv. comm.), nor are there any conspicuous features in IRAS maps. The apparent polar plane in Fig. 2 may be entirely fortuitous and is highly uncertain due to poorly known distances. Taken at face value the apparent alignment of dSphs and dIrrs with a polar plane may imply that these galaxies are on polar orbits and might be relics of a massive parent galaxy that merged long ago with M31.

\section{The Local Group: A Statistical Approach}

We seek to use the Local Group as a test case for the link between star formation history and spatial distribution (and potential interactions) of its members. Due to lack of orbital information, lack of accurate distances, and incomplete data for star formation histories we resort to a statistical approach. This approach indicates for instance that a larger number of star formation episodes is expected for satellites near M31's equatorial plane (see previous section, Fig. 1) as is indeed observed for M31's dEs.

A number of Local Group galaxies are actively forming stars even though they are relatively isolated (distant dIrrs). Other actively star-forming galaxies such as the Magellanic Clouds are strongly interacting with each other and the Milky Way. Many of the Local Group dwarfs show enhanced star formation at intermediate and old ages, either in short distinct or extended episodes. 
We have established a comprehensive database of star formation and enrichment histories (Grebel 1997) that is continuously being updated. Proper motion measurements (still affected by large errors) exist for six nearby dwarfs so far. Radial velocities (based on $\mathrm{H}$ I or stars) are available for most dIrrs. One velocity component, distance, and mass estimates are insufficient to constrain orbits. We are therefore using a statistical approach that compares star formation rates and times with present-day position and distances in the Local Group.

The most important bias in these studies is the incompleteness of detected star formation episodes. Young populations (if present) can be age-dated with great accuracy, but our age/time resolution decreases logarithmically with increasing age. The oldest populations are poorly known even in nearby dwarfs. With increasing distance crowding becomes a significant problem, preventing age dating of older populations and often even their detection.

Fig. 3 shows an example for our statistical approach: The average number of star formation episodes $\left\langle\mathrm{N}_{\mathrm{SF}}\right\rangle$ for galaxies with more than one detected episode is plotted versus distance from the nearest spiral for episodes that started earlier (right) and later (left) than $7.5 \mathrm{Gyr}$ ago. The closer $(<1 \mathrm{Mpc}$ ) satellites (mostly dSphs) had the largest number of star formation episodes earlier than 7.5 Gyr ago, whereas more recent episodes are more frequent in galaxies at distances $>1 \mathrm{Mpc}$ (mostly dIrrs). This may in part be a consequence of the ram-pressure/tidal stripping scenario suggested by van den Bergh (1994). The increased $\left\langle\mathrm{N}_{\mathrm{SF}}\right\rangle$ at closer distances to the large spirals at early times may reflect the potential influence of interactions during their stay in the Local Group. When biases are neglected it seems that the $\left\langle\mathrm{N}_{\mathrm{SF}}\right\rangle$ remained fairly constant for galaxies at distances $>1 \mathrm{Mpc}$, though the aforementioned incompleteness is likely to increase that number at earlier look-back times. Other preliminary results include a possible correlation between increased star formation rates and galaxy-galaxy separations on scales of a crossing time, another possible indication of the impact of interactions.

Acknowledgements. EKG gratefully acknowledges support by D. Zaritsky through NASA LTSA grant NAG-5-3501. TSK is supported by the NASA ATP grant (NAG 5-3061). WB acknowledges support under NASA/STScI grant GO7370.01-96A.

\section{References}

Freedman, W.L., Madore, B.F. 1990, ApJ, 365, 186

Grebel, E.K. 1997, Reviews in Modern Astronomy, 10, 29

Grebel, E.K. 1998, in: Galaxy Interactions at Low and High Redshift, IAU Symp. 186, Dordrecht: Kluwer

Lynden-Bell, D., Lynden-Bell, R.M. 1995, MNRAS, 275, 429

Majewski, S.R. 1994, ApJ, 413, 17

van den Bergh, S. 1994, ApJ, 428, 617

van den Bergh, S. 1998, AJ, 116, 1688

Zaritsky, D., Smith, R., Frenk, C.S., White, S.D.M. 1997, ApJ, 478, L53 